Abstract 24 Table 1 Breakdown of heart failure population according to a contemporary diagnostic framework and utilising different LVEF thresholds for HEREF

\begin{tabular}{|c|c|c|c|c|c|c|c|c|c|}
\hline $\begin{array}{l}\text { LVEF } \\
\text { threshold } \\
\text { for HFREF }\end{array}$ & & Total & HFREF & HFPEF & HFNMSD & $\begin{array}{l}\text { HF Alt } \\
\text { cause }\end{array}$ & RHF & $\begin{array}{l}\text { HFPEF } \\
\text { and } \\
\text { HFNMSD }\end{array}$ & $\begin{array}{l}\text { Ratio of } \\
\text { HFREF to } \\
\text { HFPEF }\end{array}$ \\
\hline \multirow[t]{2}{*}{ LVEF $<55 \%$} & Number & 245 & 180 & 35 & 14 & 13 & 3 & 49 & $5.2: 1$ \\
\hline & $\%$ of total & $100 \%$ & $73 \%$ & $14 \%$ & $5.7 \%$ & $5.3 \%$ & $1.2 \%$ & $20 \%$ & \\
\hline \multirow[t]{2}{*}{ LVEF $<50 \%$} & Number & 245 & 163 & 52 & 14 & 13 & 3 & 66 & $3.2: 1$ \\
\hline & $\%$ of total & $100 \%$ & $67 \%$ & $21 \%$ & $5.7 \%$ & $5.3 \%$ & $1.2 \%$ & $27 \%$ & \\
\hline \multirow[t]{2}{*}{ LVEF $<45 \%$} & Number & 245 & 141 & 74 & 14 & 13 & 3 & 88 & $1.9: 1$ \\
\hline & $\%$ of total & $100 \%$ & $58 \%$ & $30 \%$ & $5.7 \%$ & $5.3 \%$ & $1.2 \%$ & $36 \%$ & \\
\hline \multirow[t]{2}{*}{ LVEF $<40 \%$} & Number & 245 & 123 & 92 & 14 & 13 & 3 & 106 & $1.3: 1$ \\
\hline & $\%$ of total & $100 \%$ & $50 \%$ & $\mathbf{3 8} \%$ & $5.7 \%$ & $5.3 \%$ & $1.2 \%$ & $43 \%$ & \\
\hline
\end{tabular}

LVEF, Left ventricular ejection fraction; HFREF, Heart failure with reduced ejection fraction; HFPEF, Heart failure with preserved ejection fraction; HFNMSD, Heart failure with no major structural disease; HF Alt cause, Heart failure due to an alternative cause; RHF, Right heart failure.

with HFPEF have a definite reduction in LVEF that could account for their presentation. A requirement for elevated LV diastolic pressures or elevated biomarkers +/-diastolic dysfunction to justify a diagnosis of HFPEF resulted in a number of individuals being reclassified as having no major structural heart disease. Subset analysis of the 25 patients that maintained a diagnosis of HFPEF following a more detailed echo and MRI showed that $76 \%$ had convincing markers of LV systolic dysfunction despite a normal LVEF.

Conclusions Variable diagnostic boundaries mean some definitions of HFPEF incorporate more people with subtly reduced LVEF than others. A set of positive criteria for the diagnosis of HFPEF remains challenging and resting echocardiographic measurements have significant limitations. Currently, no universal diagnostic pathway has been agreed but our framework and analysis help better understand the HFPEF phenomenon.

\section{IN HEART FAILURE WITH PRESERVED EJECTION FRACTION (HFPEF), CARDIOVASCULAR MAGNETIC RESONANCE IMAGING (CMR) DETECTS NEW, ALTERNATIVE DIAGNOSES WHICH CARRY PROGNOSTIC SIGNIFICANCE}

${ }^{1}$ Prathap Kanagala* ${ }^{2}$ Adrian Cheng, ${ }^{3}$ John McAdam, ${ }^{3}$ Anna-Marie Marsh, ${ }^{3}$ lain Squire ${ }^{3}$ Leong Ng, ${ }^{3}$ Gerry McCann. ${ }^{1}$ Biomedical Research Unit, Leicester; ${ }^{2}$ Kettering Hospital; ${ }^{3}$ Biomedical Research Unit; *Presenting Author

\subsection{6/heartjnl-2016-309890.25}

Introduction Heart failure with preserved ejection fraction (HFPEF) carries poor prognosis and definitive therapies are lacking. Transthoracic echocardiography (TTE) remains the primary diagnostic modality in HFPEF. We aimed to evaluate the diagnostic and prognostic utility of cardiovascular magnetic resonance (CMR) in HFPEF.

Methods Patients were recruited as part of Developing Imaging And plasMa biOmarkers iN Describing-HFPEF (DIAMOND-HFPEF): a prospective, single-centre study. Inclusion criteria were: clinical or radiographic evidence of heart failure (HF) and ejection fraction $>50 \%$ on TTE. Exclusion criteria were: myocardial infarction (MI) in the preceding 6 months, suspected or confirmed cardiomyopathy, constrictive pericarditis, non-cardiovascular life expectancy $<6$ months and severe valve/ lung/ renal disease. The CMR protocol included cine, adenosine stress/rest perfusion and late gadolinium enhancement imaging on a 3-Tesla scanner. Both TTE and CMR were performed during the index study visit and reported independently. Follow-up outcome data was collected at a minimum of 6 months post-enrollment for the primary endpoint (death and/or re-hospitalisation with HF).

Results 154 patients (mean age $72.4 \pm 10.0$ years, 51\% Male) underwent both CMR and TTE. CMR detected the following previously unknown diagnoses (total $n=42$ ): significant coronary artery disease ( $\mathrm{n}=16$, including 14 with 'silent'), microvascular dysfunction ( $\mathrm{n}=11$ ), hypertrophic cardiomyopathy $(\mathrm{n}=10)$ and constrictive pericarditis $(\mathrm{n}=5)$. During followup (median $=623$ days, interquartile range 455 - 753), there were 53 primary outcome events. Kaplan-Meier survival analysis (see Figure 1) revealed worse outcomes in the 'new diagnoses group' (Log Rank test $\mathrm{p}=0.046$ ). In a multivariate Cox Regression model comprising significant independent predictors during univariate analysis (diastolic blood pressure, NHYA class, urea, eGFR and log BNP), the 'new diagnoses

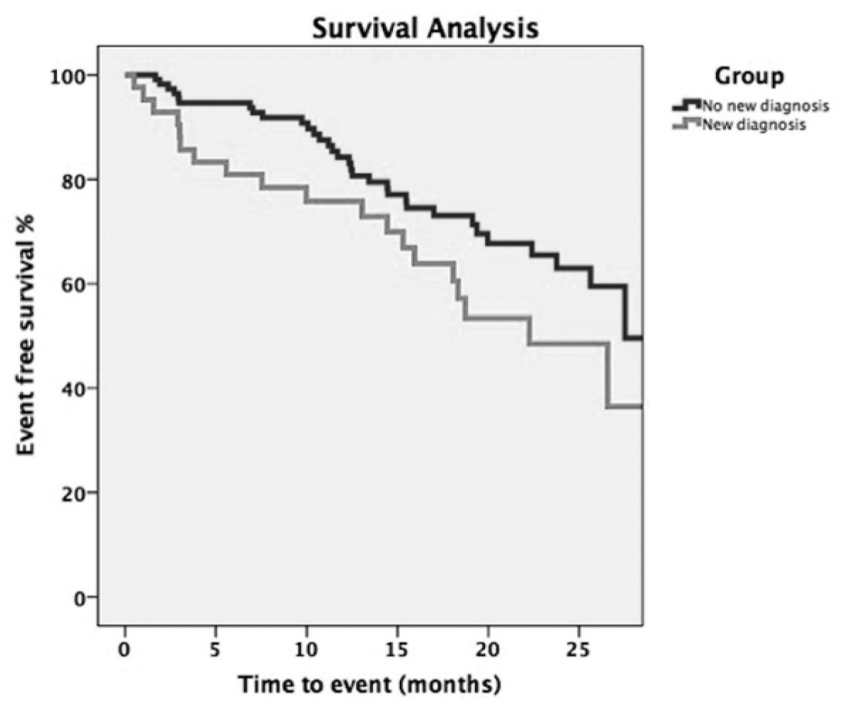

Abstract 25 Figure 1 


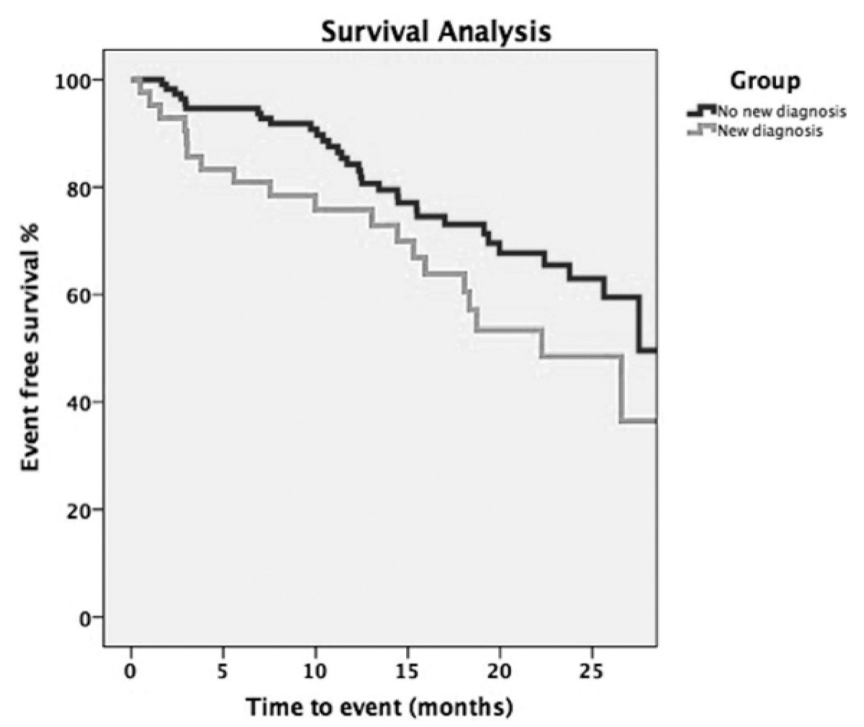

\section{Abstract 25 Figure 2}

group' (hazard ratio [HR]: 1.917; 95\% confidence interval [CI]: 1.064 to $3.454 ; \mathrm{p}=0.03$ ) remained a significant independent predictor of primary outcomes (see Figure 2).

Conclusion In HFPEF, CMR identifies previously unknown pathologies in a significant minority ( $>25 \%)$. This group of 'new diagnoses' is associated with worse outcomes and is an independent predictor of death and/or re-hospitalisation with HF.

\section{Interventional Cardiology}

\section{EFFECT OF AGE AND GENDER ON OUTCOMES FOLLOWING PRIMARY PERCUTANEOUS CORONARY INTERVENTION FOR ST-ELEVATION MYOCARDIAL INFARCTION IN A UK TERTIARY CENTRE}

${ }^{1}$ Arvindra Krishnamurthy*, ${ }^{1}$ Kathryn Somers, ${ }^{1}$ Natalie Burton-Wood, ${ }^{1}$ Michelle Anderson, ${ }^{1}$ Charlotte Harland, ${ }^{2}$ Claire Keeble, ${ }^{1} J a m e s$ McLenachan, 'Jonathan Blaxill, ${ }^{1}$ Christopher J Malkin, 'Daniel J Blackman, 'Stephen Wheatcroft, ' John P Greenwood. 'Leeds Teaching Hospitals NHS Trust; ${ }^{2}$ University of Leeds; *Presenting Author

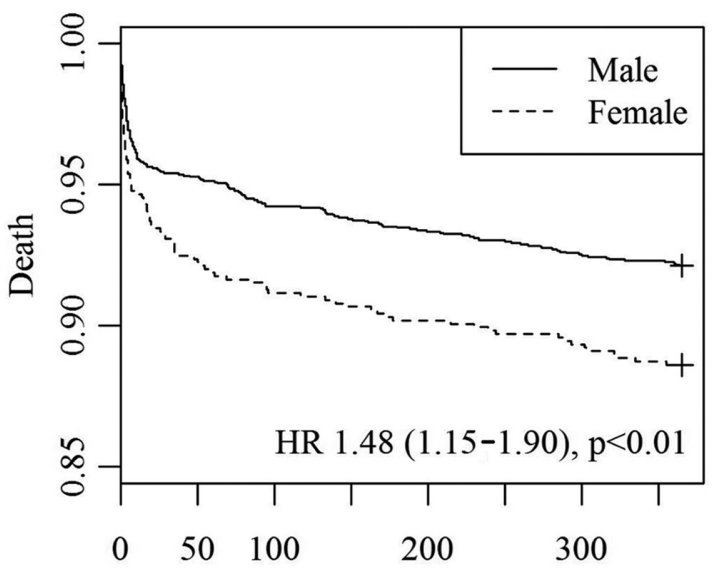

Number of days since symptom onset

\begin{tabular}{lllll} 
Abstract 25 Table 1 & & & \\
\hline & $\begin{array}{l}\text { Univariable, HR } \\
(95 \% \mathrm{Cl})\end{array}$ & $\begin{array}{l}\mathbf{p} \\
\text { Value }\end{array}$ & $\begin{array}{l}\text { Multivariable, HR } \\
(95 \% \mathrm{Cl})\end{array}$ & $\begin{array}{l}\mathbf{p} \\
\text { Value }\end{array}$ \\
\hline Age & $1.014(0.985-1.045)$ & 0.336 & & \\
Gender & $1.480(0.843-2.598)$ & 0.172 & & \\
$\begin{array}{l}\text { Systolic Blood } \\
\text { Pressure }\end{array}$ & $0.995(0.983-1.007)$ & 0.377 & & \\
Diastolic Blood & $0.973(0.950-0.997)$ & 0.026 & $0.991(0.967-1.016)$ & 0.484 \\
Pressure & & & & \\
NYHA III/IV & $1.802(1.024-3.171)$ & 0.041 & $1.552(0.833-2.893)$ & 0.166 \\
Urea & $1.086(1.022-1.153)$ & 0.007 & $1.104(1.007-1.211)$ & 0.035 \\
eGFR & $0.987(0.973-1.001)$ & 0.069 & $1.009(0.989-1.030)$ & 0.369 \\
ZLog BNP pmol/I & $1.471(1.079-2.006)$ & 0.015 & $1.442(1.032-2.015)$ & 0.032 \\
New Diagnoses & $1.752(0.999-3.071)$ & 0.050 & $1.917(1.064-3.454)$ & 0.030 \\
Group & & & & \\
\hline
\end{tabular}

Introduction Techniques and pharmacotherapy for Primary Percutaneous Coronary Intervention (PPCI) continue to evolve rapidly. The West Yorkshire PPCI outcome study was established to identify important clinical and procedural variables that may impact on patient outcomes following PPCI for ST-Elevation Myocardial Infarction (STEMI). We sought to clarify the influence of age and gender on major adverse cardiovascular events (MACE), which have been variably reported, in a large consecutive patient series.

Methods Retrospective analysis of 3049 consecutive patients who underwent PPCI for STEMI between 1-1-2009 and 3112-2011 at Leeds General Infirmary. Minimum $12 \mathrm{~m}$ clinical follow-up data were collected for all; MACE was defined as total mortality, myocardial infarction and unplanned revascularisation. Unadjusted and adjusted analyses for total mortality and MACE were performed with Cox proportional hazards models for male $(n=2223)$ and female $(n=826)$ patients and for three age tertiles - under 60yrs (Group 1, $\mathrm{n}=1276$ ), 60 to $79 \mathrm{yrs}$ (Group 2, $\mathrm{n}=1391$ ) and $80 \mathrm{yrs}$ and above (Group 3, $\mathrm{n}=382$ ).

Results Females (mean $68 \pm 13 \mathrm{yrs}$ ) had significantly higher rates of total mortality and MACE compared to males (mean $61 \pm 13$ yrs) (Table 1, Figure 1). However, when adjusted for age, there was no statistically significant difference in total

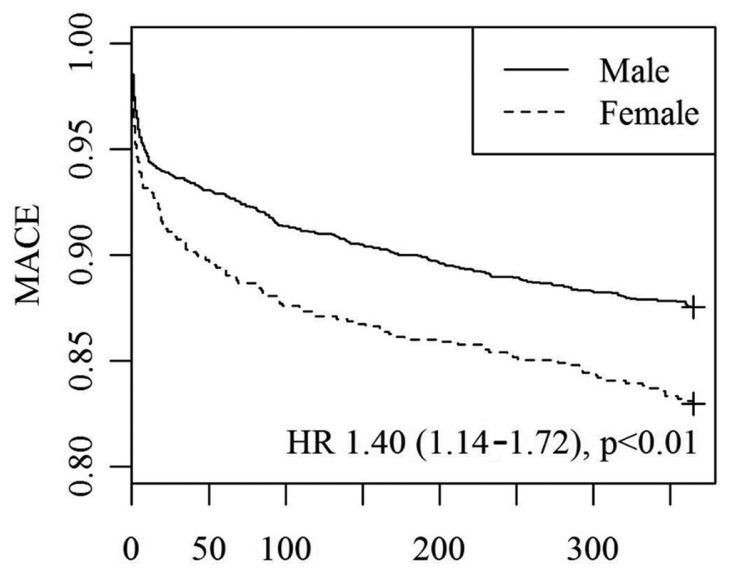

Number of days since symptom onset

Abstract 26 Figure 1 Kaplan-Meier survival curves for total mortality and MACE in females and males 